\title{
SOCIOCULTURAL IDEALIZATION OF THIN FEMALE BODY SHAPES: AN INTRODUCTION TO THE SPECIAL ISSUE ON BODY IMAGE AND EATING DISORDERS
}

\author{
JANET POLIVY AND C. PETER HERMAN \\ University of Toronto
}

Sociocultural pressure on women to be thin has been blamed for the development of eating disorders. Despite decades of research, however, it is still not clear why a few women exposed to these pressures develop eating disorders, but most women in the society do not. The media are often blamed for spreading the message that women must be thin, and for making women feel badly about themselves. This view seems overly simplistic, however, ignoring the fact that women voluntarily expose themselves to thin media images, that such exposure can actually be pleasurable, and that most women exposed to this message do not develop eating disorders. The sociocultural model of eating disorders needs further study and refinement, and the studies in this special issue represent steps in that process.

Why do eating disorders occur so much more often in women than in men? Sociocultural factors have recently become the prime suspect (e.g., Polivy \& Herman, 2002). We know that eating disorders are most likely to occur in societies where food is abundantly available, but where the idealized body shape for women is slender and tubular rather than rounded or curvaceous. The idealization of thinness (and a corresponding derogation of fatness) has been much more intense for females than for males over the last few decades (Striegel-Moore, 1997). Although living in a society where food is abundant and female thinness is idealized does not inevitably produce an eating disorder-most of the women in such a society after all, will not become eating disordered-many women will internalize the message and strive to be thin. A vulnerable few will go further and develop real eating pathololgy (Polivy \& Herman, 2002).

Despite decades of research into the sociocultural model of eating disorders, we still do not understand how such sociocultural influences produce disordered eating in any given individual (or why a similar 
person in the same cultural milieu does not become disordered). Clearly, though, one source of vulnerability lies in a woman's body image. To the extent that a woman's self-image is challenged or threatened by an unattainable ideal of an impossibly thin female physique, she may well become susceptible to disruption of her self-regard, and may be more likely to develop an eating disorder. In short, the sociocultural model argues that exposure to idealized media images (a) makes women feel bad about themselves and (b) impels women to undertake the sort of "remedial" eating patterns that easily and often deteriorate into eating disorders.

It is clear, though, that in order for sociocultural pressures to have this sort of impact on large numbers of women, the emphasis on thinness as an ideal must be communicated to the vast majority of women in the culture. One source of widespread communication of the message that thinness is desirable (and conversely that fatness is not) is the media-magazines, television, movies, etc. Ubiquitous ultra-slim models, actresses, and other media figures, and the almost complete absence of heavier women in the media, send a clear message to Western women that to be attractive, desirable, and successful, they must be thin. Endless repetition presumably increases the likelihood that the message will become internalized and have an impact on women's behavior. Nevertheless, there is some variation in the extent to which women internalize our culture's valuation of slimness, and it is the extent to which the valuation is internalized that predicts eating-disorder-related pathology such as body dissatisfaction, drive for thinness, and some bulimic characteristics (Stormer \& Thompson 1996; Stice, 2001). Do the women who do not internalize the message-or who internalize it less than others do-have less exposure to media messages, or are they somehow less susceptible to those messages, even if they experience them to the same extent? This question is only one of many raised (but rarely addressed) by the sociocultural model.

One clear assumption of the sociocultural model is that women feel bad about themselves when they are bombarded by the message that they are not thin enough. Many studies have shown that exposure to thin media images leads to body dissatisfaction, lowered self-esteem, and negative emotions in young women (Groesz, Levine, \& Murnen, 2002). Several of the articles in this issue examine reactions to exposure to media images. Hausenblas, Janelle, Gardner, and Focht (2004) examine the effects of viewing images of a thin model or of oneself; in addition, these researchers consider Drive for Thinness as a possible moderating variable. Agliata and Tantleff-Dunn (2004) extend the research to males and examine body dissatisfaction and depression in males after exposure to body-related advertisements. Halliwell and Dittmar (2004) 
separate the normally confounded variables of thinness and attractiveness in their comparison of the effectiveness of normal-weight and thin advertising models, questioning the actual effectiveness of using thin models in advertising for women. Tiggemann and McGill (2004) explore a potential explanation for the negative impact of thin models on women's self-image and mood. The current volume thus extends in several directions our understanding of the psychological impact of exposure to media images promoting the thin ideal.

One issue not specifically examined in this volume, however, is the question of why women expose themselves to media images that make them feel anxious, depressed, self-critical, and generally miserable about their own bodies. Why do women buy fashion magazines featuring thin models if seeing those models makes them so uncomfortable? Several studies shed some light on this apparent paradox.

First of all, a number of studies do not find universal negative reactions to media-image exposure (e.g., Champion \& Furnham, 1999; Cusumano \& Thompson, 1997; Joshi, Herman, \& Polivy, in press; Mills, Polivy, Herman, \& Tiggemann, 2002). Mills et al., for instance, demonstrated that the effect of viewing media images of thin females is negative only under certain conditions. When students were exposed to ads containing thin models, and were led to compare themselves to the ads by answering questions about themselves and their feelings, they indeed felt worse about themselves. (This phenomenon is addressed directly in the article in the present volume by Tiggemann and McGill, in their study of social comparison effects.) In another experimental condition in the Mills et al. study, students were asked merely to view the images and rate their effectiveness as advertising, and were then given other tasks and finally asked about themselves as part of a supposedly separate study. In this condition, exposure to the thin media images actually made students feel better about their own bodies. Mills et al. attributed this result to a "thin fantasy" stimulated by the images. Presumably, young women look at the thin images in magazines and other media and fantasize about what it would be like to resemble the model, and/or are inspired to emulate the models (Joshi et al., in press; Mills et al., 2002; Trottier, Polivy, \& Herman, in preparation).

We may conclude that media images of the thin ideal can be either inspirational or distressing, depending on whether they are seen as attainable or completely out of reach. When one compares oneself directly to a slim, beautiful model, the effect may be more painful than pleasant, because it is readily apparent how little one resembles the model. However, if one is able to enter into a pleasant fantasy about how nice it would be to be like the model, and even feel energized to 
emulate her, the experience may be pleasurable. Only if we can entertain (and explain) the possibility of a pleasant reaction to thin media images can we account for the fact that women voluntarily buy fashion magazines and in other ways (e.g., films, television) choose to expose themselves to images of impossibly thin, attractive models. The message that is internalized remains the same-namely, that a thin physique is desirable. But the hedonic tone associated with this message may be more positive than negative. Women may pursue thinness more as a matter of positive reinforcement (the desire to share in the rewards of thinness) than as a matter of negative reinforcement (the desire to escape one's current unsatisfactory body). In either case, of course, the effect of exposure to idealized media images may be to promote dieting, from which it is a short step to disordered eating. And disordered eating, in certain vulnerable personalities, is a strong predictor of eating disorders.

The societal glorification of slimness and disparagement of fatness causes many, if not most, young women to become dissatisfied with their weight and shape (e.g., Cash \& Henry, 1995; Polivy \& Herman, 1987). Body dissatisfaction is often regarded as an essential precursor (and continuing accompaniment) of eating disorders (e.g., Stice, 2001). The more intense this dissatisfaction, the more likely that one will undertake attempts to lose weight, and dieting has itself been identified as another contributor to disordered eating (Polivy \& Herman, 1987). New work is still needed to understand the relations between these variables and their effects on associated behaviors. One of the articles in this special issue examines the relation of body dissatisfaction and body image to interpersonal discomfort and attachment in relationships (Cash, Theriault, \& Annis, 2004). Another attempts to identify who develops an eating disorder after exposure to society's pressure to be thin and who does not (Pelletier, Dion, \& Lévesque, 2004) raising the question of whether there are individual protective factors, along with the vulnerabilities that have been the subject of research thus far. Indeed, one of the unfortunate aspects of the sociocultural model is that it focuses too heavily on the pathological outcomes of exposure to media images, instead of reminding us regularly that most young women who are exposed to such images manage to avoid pathological outcomes. The focus on pathology makes it easy to blame the media, which always seems to be implicated when the cause of eating disorders is debated. If more attention were paid to the majority (nonpathological) outcomes, then perhaps we would be less inclined to blanket condemnations of the media and be more attentive to the factors that make young women more or less vulnerable to its messages.

Not only are we growing more aware that exposure to idealized media 
images is not in and of itself necessarily fatal to healthy psychological development, but we are increasingly forced to ask why these images are so popular. Media people are quick to note that their customers "demand" thin models, actresses, and movie stars. It is difficult to dismiss this exculpatory refrain. We do have a choice about which media we patronize, and the sales figures suggest that we incline toward slender images. Is it fair to argue that we have been brainwashed by the media to prefer the images that harm us? That argument seems a little forced. Still, we must ask at the same time where we acquired our preference for slim physiques. Presumably it is acquired from the culture, and the media must play an (important) role. The sociocultural model must contend with this dialectic: Does the media give us what we want or does it make us want what it has to give us?

Any attempt to decide the issue of which comes first, media messages or societal preferences, is bound to fail. Certainly, the tools of experimental psychology are incapable of establishing the priority of this particular chicken and egg. So what are we (researchers) to do about body image dissatisfaction, which is clearly implicated in the genesis of eating disorders, and which is clearly exacerbated by exposure to images of slim physiques that are perceived as unattainable? We suggest that the notion that media images create body dissatisfaction, which increases the probability of disordered eating, requires an overhaul. For one thing, the problem probably does not start with the media (although the media may exacerbate the problem). For another, the emotional effect of exposure to idealized images is not always aversive. Finally, even if the emotional effect is aversive, it does not inevitably lead to disordered eating. Each component of the sociocultural model as it is currently understood has an element of truth in it, but the full picture is much more complicated than the simple model that is casually invoked so often. It is incumbent on us to (a) articulate the model as clearly as possible, emphasizing that the elements of the model remain hypotheses, not facts, and (b) test the model empirically, while remaining alert to the likelihood that the simple model will have to be adjusted, repeatedly, to conform to new data and the development of new, more sophisticated hypotheses. The articles included in this special issue are representative of the energetic work currently being conducted. Let us hope that a decade or two from now, these articles (and others like them) will provide the empirical grist for a more useful and mature theory of how the media and body dissatisfaction contribute to (and reflect) the eating disorders that we currently understand so dimly. 


\section{REFERENCES}

Agliata, D., \& Tantleff-Dunn, S. (2004). The impact of media exposure on males' body image. Journal of Social and Clinical Psychology, 23(1), pp. 7-22.

Cash, T. F., \& Henry, P.E. (1995). Women's body images: The results of a national survey in the USA. Sex Roles, 33, 19-28.

Cash, T.F., Thériault, J., \& Annis, N.M. (2004). Body image in an interpersonal context: Adult attachment, fear of intimacy, and social anxiety. Journal of Social and Clinical Psychology, 23(1), pp. 89-103.

Champion, H., \& Furnham, A. (1999). The effect of the media on body satisfaction in adolescent girls. European Eating Disorders Review, 7, 213-228.

Cusumano, D.L., \& Thompson, J.K. (1997). Body image and body shape ideals in magazines: Exposure, awareness, and internalization. Sex Roles, 37, 701-721.

Groesz, L. M., Levine, M.P., \& Murnen, S.K. (2002). The effect of experimental presentation of thin media images on body satisfaction: A meta-analytic review. International Journal of Eating Disorders, 31, 1-16.

Halliwell, E., \& Dittmar, H. (2004). Does size matter? The impact of model's body size on women's body-focused anxiety and advertising effectiveness. Journal of Social and Clinical Psychology, 23(1), pp. 104-122.

Hausenblas, H.A., Janelle, C.M., Gardner, R.E., \& Focht, B.C. (2004). Viewing physique slides: Affective responses of high and low drive for thinness women. Journal of Social and Clinical Psychology, 23(1), pp. 45-60.

Joshi, R., Herman, C.P., \& Polivy, J. (In press). Self-enhancing effects of exposure to thin-body images. International Journal of Eating Disorders.

Mills, J., Polivy, J., Herman, C.P., \& Tiggemann, M. (2002). Effects of media-portrayed idealized body images on restrained and unrestrained eaters. Personality \& Social Psychology Bulletin, 28, 1687-1699.

Pelletier, L.G., Dion, S.C., \& Séguin-Lévesque, C. (2004). Can self-determination help protect women against sociocultural influences about body image and reduce their risk of experiencing bulimic symptoms? Journal of Social and Clinical Psychology, 23(1), pp. 61-88.

Polivy, J., \& Herman, C. P. (1987). The diagnosis and treatment of normal eating. Journal of Consulting \& Clinical Psychology, 55, 635-644.

Polivy, J., \& Herman, C. P. (2002). Causes of eating disorders. Annual Review of Psychology, $53,187-213$.

Stice, E. (2001). A prospective test of the dual-pathway model of bulimic pathology: Mediating effects of dieting and negative affect. Journal of Abnormal Psychology, 110, 1-12.

Stice, E., Schupak-Neuberg, E., Shaw, H.E., \& Stein, R.I. (1994). Relation of media exposure to eating disorder symptomatology: An examination of mediating mechanisms. Journal of Abnormal Psychology, 103, 836-840.

Stormer, S.M., \& Thompson, J.K. (1996). Explanations of body image disturbance: A test of maturational status, negative verbal commentary, social comparison, and sociocultural hypotheses. International Journal of Eating Disorders, 19, 193-202.

Striegel-Moore, R. (1997). Risk factors for eating disorders. Annals of the New York Academy of Sciences: Adolescent nutritional disorders: Prevention and Treatment, 817, 98-109.

Tiggemann, M., \& McGill, B. (2004). The role of social comparison in the effect of magazine advertisements on women's mood and body dissatisfaction. Journal of Social and Clinical Psychology, 23(1), pp. 23-44. 
Copyright of Journal of Social \& Clinical Psychology is the property of Guilford Publications Inc. and its content may not be copied or emailed to multiple sites or posted to a listserv without the copyright holder's express written permission. However, users may print, download, or email articles for individual use. 